\title{
ESTIMATES OF DISPERSIVE EFFECTS IN A BENT NLC MAIN LINAC*
}

\author{
M. Syphers and L. Michelotti, Fermilab, Batavia, IL 60510, USA
}

\begin{abstract}
An alternative being considered for the Next Linear Collider (NLC) is not to tunnel in a straight line but to bend the Main Linac into an arc so as to follow a gravitational equipotential. We begin here an examination of the effects that this would have on vertical dispersion, with its attendant consequences on synchrotron radiation and emittance growth by looking at two scenarios: a gentle continuous bending of the beam to follow an equipotential surface, and an introduction of sharp bends at a few sites in the linac so as to reduce the maximum sagitta produced.
\end{abstract}

\section{CONTINUAL GENTLE BENDS}

In our first scenario, the Main Linac remains as close as possible to an equipotential surface. Minimalism suggests that we try bending the beam by vertically translating already existing NLC quadrupoles, without introducing new elements or additional magnetic fields. We thus propose that steering be accomplished by precisely aligning all the quads "level" along the equipotential and then raising the vertically defocusing (D) quadrupoles to steer the beam through the centers of the vertically focusing (F) quads. ${ }^{1}$ Bending at the D quad locations will minimize the generated dispersion.

To estimate the order of magnitude of dispersion produced by such an arrangement, we calculate (a) assuming a periodic sequence of magnets while (b) neglecting the effects of acceleration [1] and (c) keeping only leading terms in the bend angle. Our results will be reasonably correct provided that upstream injection into the Main Linac is redesigned to match the new arrangement. Further details of the calculation and others discussed in this paper are documented elsewhere. [2]

Figure 1 shows the physical layout of quadrupoles and identifies the geometric parameters. It is practical to write the vertical offset of the quadrupole relative to the equipotential, $d-y_{\text {sag. }}$. In terms of the distance between quadrupoles, $L$, the local betatron phase advance per cell, $\mu$, and the radius of the earth, $R$, this offset is

$$
d-y_{\text {sag }}=\frac{L^{2}}{R}\left(\frac{1}{2}+\frac{1}{\sin (\mu / 2)}\right)
$$

To make a numerical estimate of this offset at the high energy end of the linac, we take $L \approx 19 \mathrm{~m}, \mu \approx \pi / 2$, and $R \approx 6400 \mathrm{~km}$. Eq.(1) then yields $d-y_{\mathrm{sag}} \approx 108 \mu \mathrm{m}$. The dispersion can be estimated easily using two observations:

\footnotetext{
* Work supported by the U.S. Department of Energy

${ }^{1}$ The usual convention is for "F" ("D") to indicate a horizontally focusing (defocusing ) quadrupole. We do the opposite here, because we are considering dynamics only in the vertical plane.
}

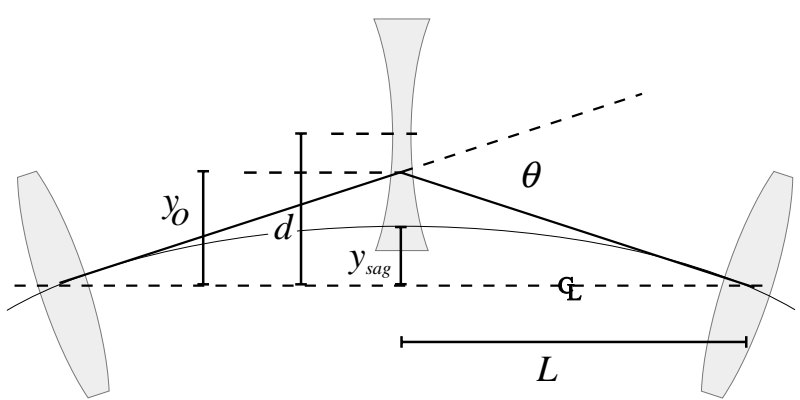

Figure 1: Description of parameters for describing the CR thin quad calculations.

(1) in passing through a thin bending magnet, the slope of the dispersion function, $D^{\prime}$, changes by an amount approximately equal to the bend angle; (2) by symmetry, the dispersion attains its maximum (minimum) value at the center of the focussing (defocussing) quadrupole. The values of the dispersion function at the thin-lens quadrupoles will be

$$
\begin{aligned}
D_{\min } & =\frac{L^{2}}{R \sin ^{2}(\mu / 2)}(1-\sin (\mu / 2)), \\
D_{\max } & =\frac{L^{2}}{R \sin ^{2}(\mu / 2)} .
\end{aligned}
$$

Using the same parameters as before, this provides the numerical estimate, at the high energy end of the linac,

$$
D_{\min }=0.032 \mathrm{~mm}, D_{\max }=0.11 \mathrm{~mm} \text {. }
$$

If we take a large $\Delta p / p \approx \Delta E / E=0.02$, because of BNS damping, and assume that the "invariant emittance" $\gamma \epsilon_{y} / \pi \approx 100 \mathrm{~nm}$ and $\beta_{y} \approx 40 \mathrm{~m}$ at a point where the electron's energy is $E=100 \mathrm{GeV}$, then $D_{\max } \cdot \frac{\Delta p}{p}=2.2 \mu \mathrm{m}$ compared to $\sigma_{y}=\sqrt{\beta_{y} \epsilon_{y} / \pi}=4.6 \mu \mathrm{m}$.

Vertical bending will produce synchrotron radiation, which, in its turn, will add to the vertical emittance of the beam. At high energy, the total energy radiated by one electron is given by the expression, ${ }^{2}$

$$
U=\int(c d t) \frac{1}{6 \pi \epsilon_{o}}\left(\frac{e}{\rho}\right)^{2} \gamma^{4},
$$

where $\rho$ is the bend radius, $\gamma$ is the relativistic $1 / \sqrt{1-(v / c)^{2}}$, and the other variables need no introduction. In terms of the electron energy, $E$, and the bend angle, $\theta$, produced by the quad over its length, $\ell$,

$$
U=\left(1.41 \times 10^{-5} \mathrm{~m} \mathrm{GeV}^{-3}\right) \cdot E^{4} \theta^{2} / \ell .
$$

\footnotetext{
${ }^{2}$ For example, see Equations 8.6 and 8.10 of Edwards and Syphers. [3]
} 
Using the same parameters as before, $\theta \approx 5.6 \mu \mathrm{rad}$; at the high energy end of the linac, $E \approx 500 \mathrm{GeV}$, and $\ell \approx 1 \mathrm{~m}$; our estimate of the total radiated energy (per electron per bend) is about $28 \mathrm{keV}$. Put another way, the ratio, $U / E \approx 6 \times 10^{-8}$. It is inconceivable that such a small fractional change in beam energy could seriously damage the emittance, but we will estimate its effect anyway. The additional invariant emittance due to synchrotron radiation is approximated as

$$
\Delta\left(\gamma \epsilon_{y} / \pi\right) \approx \frac{55}{6 \sqrt{3}} \frac{r_{e} \hbar c}{m c^{2}}\langle\mathcal{H}\rangle\left\langle\frac{1}{\rho^{2}}\right\rangle \theta \gamma^{6},
$$

where $\epsilon_{y}=\pi \sigma_{y}^{2} / \beta_{y}$ and $r_{e}$ is the classical electron radius. The quantity $\langle\mathcal{H}\rangle \approx D_{y}^{2} / \beta_{y}$ at the quadrupole location.

Plugging in the same numbers as before, estimating $\beta_{y} \approx 60 \mathrm{~m}$, and using our previous estimates for $U$ and $D_{\max }$, we obtain $\Delta\left(\gamma \epsilon_{y} / \pi\right) \approx 1.8 \times 10^{-7} \mathrm{~nm}-$ as expected, a very small number.

\section{LOCALIZED SHARP BENDS}

Although the $2 \mu \mathrm{m}$ offset of an off-momentum particle predicted in the previous section is not catastrophic, neither is it completely negligible. We will now consider eliminating it by employing the second scenario: constructing a Main Linac that is laser straight except for highly localized bends at a few, widely separated locations. These bend sites then allow us to follow the equipotential in a coarser, piecewise fashion. If we think of bending every kilometer, or so, then the bend angle should be about $160 \mu \mathrm{rad}$. We'll take this as the "canonical" value for calculations in this section.

We will proceed again in a minimalist way. In order to minimize the modification of existing lattice hardware and optics, we adopt the use of combined function magnets to both bend and focus the electron beam. Other possibilities could be considered at a later date. If we only "bend" at each of the local sites, a dispersion wave of amplitude $\sim 4 \mathrm{~mm}$ would be generated at each bend center. To match the trajectories at the end of each local bending region for particles with various momenta, the total bend angle of each region is distributed across four neighboring (combined function) dipoles. The strategy is akin to that of an $18^{\text {th }}$ century optician designing a simple focussing achromat.

The results at the lowest energy bending location are shown in Figure 2. The dashed line follows the residual dispersion, now completely contained within the $\approx 40 \mathrm{~m}$ long bending region, with maximum amplitude of about $0.6 \mathrm{~mm}$. The maximum orbit distortion of $1 \mathrm{~mm}$ is too large an offset from the central (curved) axis of the local bending magnets. They would have to be displaced so as to follow the new orbit. A few iterations of these manipulations should then converge on an acceptable design. However, the final orbit and its local residual dispersion should not be much different from what we have calculated here. For now, we simply display these results as indicating the order of magnitude of the effects.

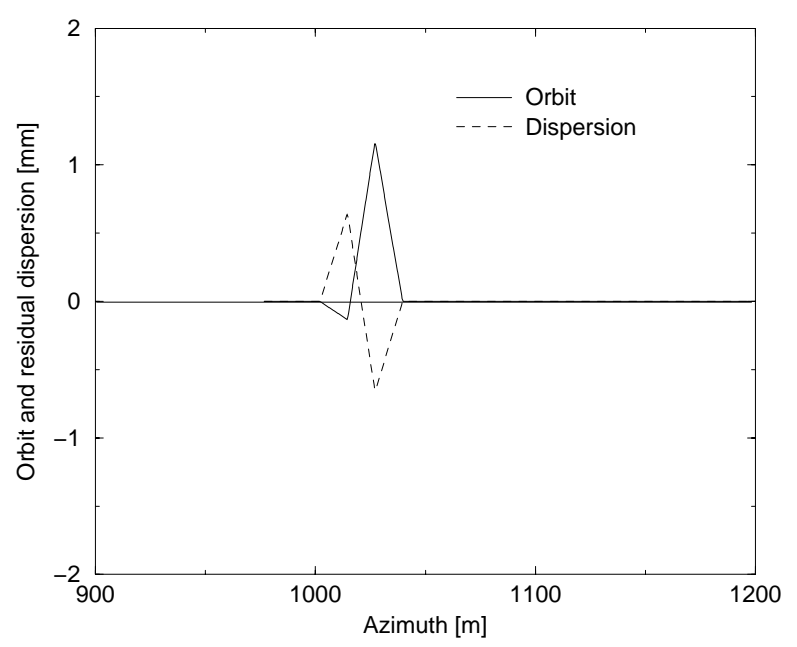

Figure 2: Orbit deviation required to zero the residual dispersion.

The distortions in the trajectory at ten locations located $\sim 1 \mathrm{~km}$ apart in the NLC are of the order 1-2 mm, with the higher displacements occuring at the higher energies. The corresponding dispersions generated along the linac are shown in Figure 3. A residual dispersion of $1 \mathrm{~mm}$ remains in the neighborhood of the bends. Again assuming that $\Delta p / p \approx 0.02$, we have $D_{\max } \cdot \frac{\Delta p}{p}=20 \mu$ m compared to $\sigma_{y}=\sqrt{\beta_{\max } \epsilon_{y} / \pi}=4.6 \mu \mathrm{m}$. This is a large increase, but it exists only near the bend sites. Away from these sites, the dispersion is (essentially) zero, and its contribution to emittance is negligible. We note in passing that an advantage of this calculation is that one can envision making it opera-

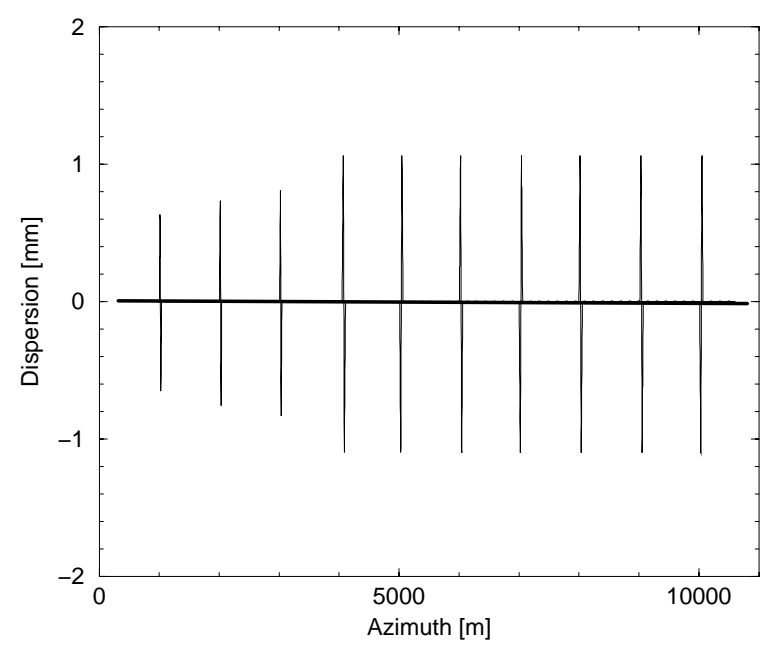

Figure 3: (a) Residual dispersion generated by the partial achromats at all ten locations along the NLC. 
tional.

Finally, we estimate the synchrotron radiation and emittance growth incurred by our second scenario, once again using Eqs.(2) and (3). The values of $\Delta\left(\gamma \epsilon_{y} / \pi\right)$ at all bend locations are plotted in Figure 4. Each site contains one dominant, very sharp bend. Its effect is most apparent near the high energy end of the linac, where the $E^{6}$ dependence becomes overwhelming. Even so, the additional $\approx 1 \mathrm{~nm}$ in invariant emittance is less than $1 \%$ of the $140 \mathrm{~nm}$ vertical emittance expected within the interaction region.

Notice that although the synchrotron radiation is rather high at the end of the linac, the ratio $U / E=49 \mathrm{MeV} / 473 \mathrm{GeV} \approx 10^{-4}$ is still a small number.

In passing, we note that some attention should be given to coherent synchrotron radiation (CSR) from individual bunches. A quick look[2] shows that the current NLC design has an aperture $a \approx 7 \mathrm{~mm}$, so CSR is forbidden, even at the high energy end of the linac, because of "shielding" from the walls of the beam pipe. However, the margin of safety is not comfortably large. It may be necessary to reexamine this issue.

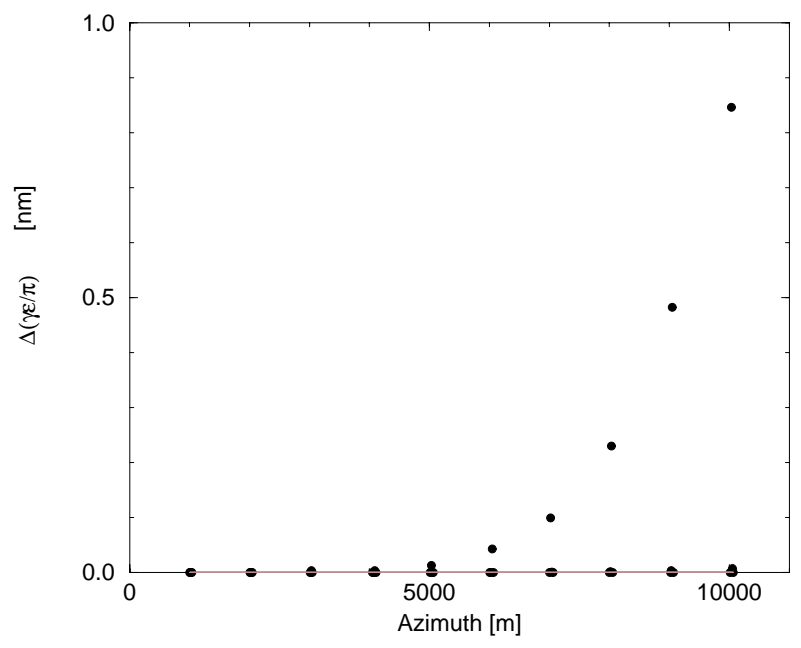

Figure 4: Emittance growth due to synchrotron radiation in sharp bends.

\section{ULTIMATE ENERGY OF “CURVED" LINAC}

While present NLC designs with beam energies in the range of a few hundred $\mathrm{GeV}$ to $1 \mathrm{TeV}$ may not be very sensitive to the curvature of the earth, there will be a practical limit to the upgraded energy of such a device. Suppose the scheme of steering the beam with offset quadrupoles is adopted. Then, at high energies, eventually the energy gain within a FODO cell will be equal to the energy lost due to synchrotron radiation as the beam is bent by the offset
Energy, emittance vs. Linac length

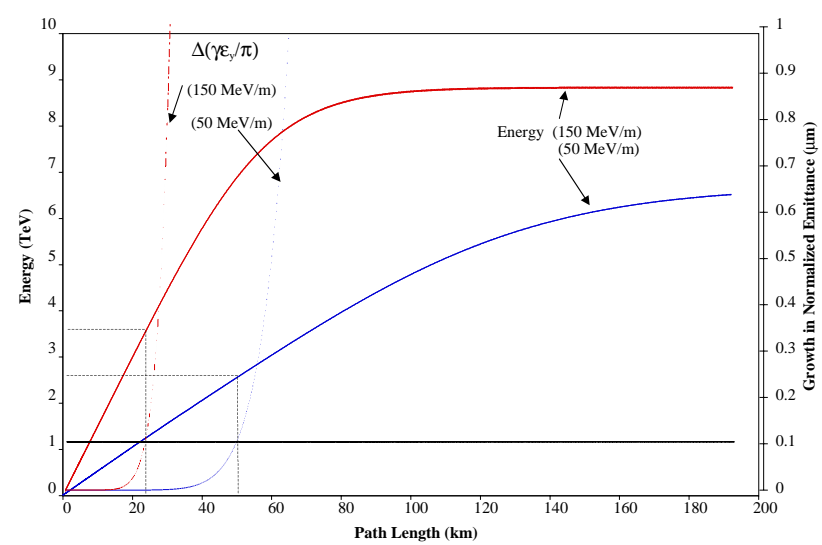

Figure 5: Beam energy and emittance growth vs.path length of linac which follows the curvature of the earth. Results with accelerating gradients of $50 \mathrm{MeV} / \mathrm{m}$ and $150 \mathrm{MeV} / \mathrm{m}$ are shown.

quadrupole. This limit can be easily written as

$$
E_{l i m}=\left(\frac{\pi}{C_{\gamma}} \frac{\ell_{q}}{L} E_{c v} R^{2}\right)^{1 / 4}
$$

where $E_{c v}$ is the energy gain per meter of the linac. As the energy is increased, both the quadrupole length (strength) and the half-cell length increase roughly proportionally. As an example, using $E_{c v}=50 \mathrm{MeV} / \mathrm{m}$, and $\ell_{q} / L=0.025$, then we get $E_{\text {lim }} \approx 6.5 \mathrm{TeV}$.

This conclusion is illustrated in Figure 5. The emittance growth due to synchrotron radiation is also plotted, showing that a growth of $\Delta\left(\gamma \epsilon_{y} / \pi\right)=100 \mathrm{~nm}$ (roughly equal to the NLC nominal design value at collision) occurs well before the final energy is reached, with the emittance growth being a steep function of energy $\left(\sim \gamma^{6}\right)$. For $50 \mathrm{MeV} / \mathrm{m}$, the practical limit of a curved linac may be only about 2$3 \mathrm{TeV}$ per beam, which would have a length of about 50 $\mathrm{km}$. For $150 \mathrm{MeV} / \mathrm{m}$, the limits are 3-4 TeV per beam over about $20 \mathrm{~km}$. Note that a "laser straight" linear collider with $20 \mathrm{~km}$ per linac, and a lengthy interaction region, with its two ends near the surface of the earth would have its collision point located roughly $50 \mathrm{~m}$ below the surface.

\section{REFERENCES}

[1] Leo Michelotti. A two-parameter accelerating FODO cell. FERMILAB-FN-688, January 2000.

[2] L. Michelotti and M. Syphers. Estimates of dispersive effects in a bent NLC main linac. FERMILAB-FN-690, May 2000 .

[3] D. A. Edwards and M. J. Syphers. An Introduction to the Physics of High Energy Accelerators. John Wiley \& Sons, New York, 1993. 